1 Hacettepe Journal of Mathematics and Statistics

$\bigcap$ Volume 46 (1) (2017), 149-162

\title{
Ideal convergence in 2-fuzzy 2-normed spaces
}

\author{
Mohammad H.M. Rashid * and Ljubiša D.R. Kočinac ${ }^{\dagger}$
}

\begin{abstract}
In this paper we introduce the notion of J-convergence and JCauchyness of sequences in 2-fuzzy 2-normed spaces and established some basic results related to these notions. Further, we define J-limit and J-cluster points of a sequence in a 2-fuzzy 2-normed linear space and investigate the relations between these concepts.
\end{abstract}

Keywords: 2-fuzzy 2-norm, ideal convergence, ideal Cauchy sequence

2000 AMS Classification: Primary 40A35; Secondary 46A70, 54A20, 54A40

\section{Introduction}

Convergence (of sequences) is one of the basic and most important concepts in mathematics. It was generalized in several directions.

The notion of statistical convergence of sequences of real numbers was introduced independently by H. Fast [20] and H. Steinhaus [42], although the first idea of statistical convergence, under the name almost convergence, have appeared in 1935 in the first edition of the famous Zygmund's monograph [46]. It is based on the notion of asymptotic density of a subset of the set $\mathbb{N}$ of natural numbers. For $A \subset \mathbb{N}$ and $n \in \mathbb{N}$, let $A(n):=\{k \in A: k \leq n\}$ and let $|A(n)|$ denote cardinality of $A(n)$. The asymptotic (or natural) density of $A$ is defined by

$$
\delta(A)=\lim _{n \rightarrow \infty} \frac{|A(n)|}{n} .
$$

\footnotetext{
*Department of Mathematics \& Statistics, Faculty of Science, P.O.Box 7, Mu'tah University, Al-Karak, Jordan

Email : malik_okasha@yahoo.com

${ }^{\dagger}$ Faculty of Sciences and Mathematics, University of Niš, P.O. Box 224, Višegradska 33, 18000 Niš, Serbia

Email : lkocinac@gmail.com Corresponding author.

Doi : 10.15672/HJMS.2016.406
} 
Statistical convergence has many applications in different fields of mathematics (see, for example, $[17,22]$ and references therein). Let us mention that statistical convergence in function spaces was studied in $[7,11]$.

In 1970, Bernstein [8] introduced convergence of sequences with respect to a filter $\mathcal{F}$ on $\mathbb{N}$. Using the concept of an ideal, Kostyrko et al. [30] (see also [31]) introduced the notion of ideal convergence which is a common generalization of ordinary convergence and statistical convergence. The ideal convergence provides a general framework to study the properties of various types of convergence. Let $S$ be a non empty set. Then a family of sets $\mathcal{J} \subset 2^{S}\left(2^{S}\right.$ is the power set of $\left.S\right)$ is said to be an ideal on $S$ if for each $A, B \in \mathcal{J}$ we have $A \cup B \in \mathcal{J}$, and for each $A \in \mathcal{J}$ and each $B \subset A$, we have $B \in \mathcal{J}$. A non empty family of sets $\mathcal{F} \subset 2^{S}$ is said to be filter on $S$ if $\emptyset \notin \mathcal{F}$, for each $A, B \in \mathcal{F}$ we have $A \cap B \in \mathcal{F}$ and for each $A \in \mathcal{F}$ and each $B \supset A$, we have $B \in \mathcal{F}$. An ideal $\mathcal{J}$ on $S$ is called non-trivial if $\mathcal{J} \neq \emptyset$ and $S \notin \mathcal{J}$. It is clear that $\mathcal{J} \subset 2^{S}$ is an non-trivial ideal on $S$ if and only if $\mathcal{F}=\mathcal{F}(\mathcal{J})=:\{S \backslash A: A \in \mathcal{J}\}$ is a filter on $S$. A non-trivial ideal $\mathcal{J} \subset 2^{S}$ is called an admissible ideal if $\mathcal{J} \supset\{\{x\}: x \in S\}$. In this paper we consider the case $S=\mathbb{N}$. An admissible ideal $\mathcal{J} \subset 2^{\mathbb{N}}$ is said to have the property (AP) $[13,30]$ if for any sequence $\left\{A_{1}, A_{2}, \cdots\right\}$ of mutually disjoint sets of $\mathcal{J}$, there is a sequence $\left\{B_{1}, B_{2}, \cdots\right\}$ of subsets of $\mathbb{N}$ such that each symmetric difference $A_{i} \Delta B_{i}$ $(i=1,2, \cdots)$ is finite and $\bigcup_{i=1}^{\infty} B_{i} \in \mathcal{J}$.

We will need the following two lemmas concerning ideals with property (AP).

1.1. Lemma. ([30]) Let $\left\{A_{i}\right\}_{i=1}^{\infty}$ be a countable collection of subsets of $\mathbb{N}$ such that $A_{i} \in \mathcal{F}(\mathcal{J})$ for each $i$, where $\mathcal{J}$ is an admissible ideal with the property (AP). Then there exists a set $A \subset \mathbb{N}$ such that $A \in \mathcal{F}(\mathcal{J})$ and the set $A \backslash A_{i}$ is finite for all $i$.

1.2. Lemma. ([30]) Let $\mathcal{J} \subset 2^{\mathbb{N}}$ be an admissible ideal with the property (AP) and $(X, \rho)$ be a metric space. Then $\mathcal{J}-\lim x_{k}=x_{0}$ if and only if there exists a set $P \in \mathcal{F}(\mathcal{J}), P=\left\{p_{1}<p_{2}<\cdots<p_{k}<\cdots\right\}$ such that $\lim _{k \rightarrow \infty} x_{p_{k}}=x_{0}$.

On the other hand, the fuzzy theory has emerged as one of the most active area of research in many branches of mathematics and engineering. This new theory was introduced by Zadeh [44] in 1965 and since then a large number of research papers have appeared by using the concept of fuzzy sets/numbers and fuzzification of many classical theories has also been made.

The idea of fuzzy norm was initiated by Katsaras [29]. Felbin [21] defined a fuzzy norm on a linear space whose associated fuzzy metric is of Kaleva and Seikkala type [25]. Cheng and Mordeson [12] introduced an idea of a fuzzy norm on a linear space whose associated metric is Kramosil and Michalek type [32]. Bag and Samanta in [4] gave a definition of a fuzzy norm in such a manner that the corresponding fuzzy metric is of Kramosil and Michalek type [32]. They also studied some properties of the fuzzy norm in [5] and [6]. Bag and Samanta discussed the notions of convergent sequence and Cauchy sequence in fuzzy normed linear space in [4]. They also made in [6] a comparative study of the fuzzy norms defined by Katsaras [32], Felbin [21], and Bag and Samanta [4]. The concept of 2-normed spaces was initially introduced 
by Gähler [19] in the 1960s. Since then, this concept has been studied by many authors (see, for instance, $[4,9,10,14,18,37]$ ).

Karakuş et al. [26] defined statistical convergence in intuitionistic fuzzy normed spaces and Mursaleen et al. [34] investigated statistical convergence of double sequences in intuitionistic fuzzy normed spaces. Quite recently, in [27, 28], Karakaya et al defined and studied statistical and ideal convergence of sequences of functions in intuitionistic fuzzy normed spaces. The concept of statistical limit inferior and limit superior of sequences of fuzzy numbers found in $[2,3]$. Let us mention that statistical and ideal convergence have been studied in fuzzy context (relations with fuzzy numbers and fuzzy normed linear spaces) in many papers (see, for example, $[1,23,24,33,36,38-41])$.

In this paper we investigate ideal convergence in the fuzzy settings, more precisely in 2-fuzzy 2-normed spaces. The paper is organized as follows: In the second section, we present some preliminary definitions and results related to fuzzy normed spaces and 2-fuzzy 2-normed spaces. In the third section, we introduce the notion of $\mathcal{J}$-convergent sequence and $\mathcal{J}^{*}$-convergence in a 2 -fuzzy 2 -normed space and some basic results are obtained. In fourth section, we introduce the notion of $\mathcal{J}$-Cauchy and $\mathcal{J}^{*}$-Cauchy sequences in a 2-fuzzy 2-normed space. In Section 5 the concepts of J-limit points and J-cluster points of a sequence in a 2-fuzzy 2-normed space are defined and relations between these concepts are investigated.

\section{Definitions and preliminaries}

By $\mathbb{R}$ we denote the set of real numbers. All linear spaces are assumed to be over $\mathbb{R}$.

For the sake of completeness, we reproduce some definitions due to Gähler [19], Bag and Samanta [4], Somasundaram and Beaula [43], and Zhang [45].

2.1. Definition. ([19]) Let $X$ be a real linear space of dimension $s$, where $2 \leq$

$s<\infty$. A 2-norm on $X$ is a function $\|.,\|:. X \times X \rightarrow \mathbb{R}$ which satisfies

(i) $\|x, y\|=0$ if and only if $x$ and $y$ are linearly dependent;

(ii) $\|x, y\|=\|y, x\|$ for all $x, y \in X$;

(iii) $\|c x, y\|=|c|\|x, y\|$ for all $x, y \in X$ and $c \in \mathbb{R}$;

(iv) $\|x+y, z\| \leq\|x, z\|+\|y, z\|$ for all $x, y, z \in X$.

The pair $(X,\|.,\|$.$) is then called a 2-normed space.$

An example of a 2-normed space is the set $X=\mathbb{R}^{2}$ equipped with the 2-norm

$$
\|x, y\|=\left|x_{1} y_{2}-x_{2} y_{1}\right|, x=\left(x_{1}, x_{2}\right), y=\left(y_{1}, y_{2}\right),
$$

i.e. $\|x, y\|$ is the area of the parallelogram spanned by the vectors $x$ and $y$.

2.2. Definition. ([4]) Let $X$ be a linear space over $\mathbb{R}$. A fuzzy subset $N$ of $X \times \mathbb{R}$ is called a fuzzy norm on $X$ if for all $x, y \in X$ and $c \in \mathbb{R}$.

(FN1) For all $t \in \mathbb{R}$ with $t \leq 0, N(x, t)=0$;

(FN2) for all $t \in \mathbb{R}$ with $t>0, N(x, t)=1$, if and only if $x=0$;

(FN3) for all $t \in \mathbb{R}$ with $t>0, N(c x, t)=N(x, t /|c|)$, if $c \neq 0$,

(FN4) for all $s, t \in \mathbb{R}, x, y \in X, N(x+y, s+t) \geq \min \{N(x, s), N(y, t)\}$,

(FN5) $N(x,$.$) is a non decreasing function of \mathbb{R}$ and $\lim _{t \rightarrow \infty} N(x, t)=1$. 
The pair $(X, N)$ will be referred to as a fuzzy normed linear space.

The following definition is actually a decomposition theorem from [4].

2.3. Definition. ([4]) Let $(X, N)$ be a fuzzy normed linear space. Assume further that

(FN6) $N(x, t)>0$ for all $t>0$ implies $x=0$.

Define $\|x\|_{\alpha}=\inf \{t: N(x, t) \geq \alpha\}, \alpha \in(0,1)$. Then $\left\{\|\cdot\|_{\alpha}: \alpha \in(0,1)\right\}$ is an ascending family of norms on $X$ or $\alpha$-norms on $\mathrm{X}$ corresponding to the fuzzy norm on $X$.

2.4. Definition. ([45]) Let $X$ be a non-empty set and $\mathrm{F}(X)$ be the set of all fuzzy sets on $X$. For $U, V \in \mathrm{F}(X)$ and $k \in \mathbb{R}$ define

$$
U+V=\{(x+y, \lambda \wedge \mu):(x, \lambda) \in U,(y, \mu) \in V\},
$$

and $k U=\{(k x, \lambda):(x, \lambda) \in U\}$.

2.5. Definition. ([43]) A fuzzy linear space $\tilde{X}=X \times(0,1]$ over $\mathbb{R}$ where the addition and scalar multiplication operation on $X$ are defined by $(x, \lambda)+(y, \mu)=$ $(x+y, \lambda \wedge \mu), k(x, \lambda)=(k x, \lambda)$ is a fuzzy normed space if for every $(x, \lambda) \in \widetilde{X}$ there is associated a non-negative real number, $\|(x, \lambda)\|$, called the fuzzy norm of $(x, \lambda)$, in such a way that

(1) $\|(x, \lambda)\|=0 \Longleftrightarrow x=0$ the zero element of $X, \lambda \in(0,1]$,

(2) $\|k(x, \lambda)\|=|k|\|(x, \lambda)\|$ for all $(x, \lambda) \in \widetilde{X}$ and all $k \in \mathbb{R}$,

(3) $\|(x+y, \lambda+\mu)\| \leq\|(x, \lambda+\mu)\|+\|(y, \lambda+\mu)\|$ for all $(x, \lambda),(y, \mu) \in \widetilde{X}$,

(4) $\left\|\left(x, \vee_{t} \lambda_{t}\right)\right\|=\wedge_{t}\left\|\left(x, \lambda_{t}\right)\right\|$ for $\lambda_{t} \in(0,1]$.

2.6. Definition. ([43]) Let $X$ be a non-empty and $\mathrm{F}(X)$ be the set of all fuzzy sets in $X$. If $f \in \mathrm{F}(X)$, then $f=\{(x, \mu): x \in X$ and $\mu \in(0,1]\}$. Clearly $f$ is a bounded function for $|f(x)| \leq 1$. $\mathrm{F}(X)$ is a linear space over the field $\mathbb{R}$, where the addition and scalar multiplication are defined by

$$
f+g=\{(x, \mu)+(y, \nu)\}=\{(x+y, \mu \wedge \nu):(x, \mu) \in f \text { and }(y, \nu) \in g\}
$$

and

$$
k f=(k x, \mu) \text { such that }(x, \mu) \in f
$$

where $k \in \mathbb{R}$.

The linear space $\mathrm{F}(X)$ is said to be a normed space if for every $f \in \mathrm{F}(X)$ there is associated a non-negative real number $\|f\|$ called the norm of $f$ in such a way that

(1) $\|f\|=0$ if and only if $f=0$.

(2) $\|k f\|=|k|\|f\|, k \in \mathbb{R}$.

(3) $\|f+g\| \leq\|f\|+\|g\|$ for every $f, g \in \mathrm{F}(X)$.

Then $(\mathrm{F}(X),\|\cdot\|)$ is a normed linear space.

2.7. Definition. ([43]) A 2-fuzzy set on $X$ is a fuzzy set on $\mathrm{F}(X)$.

2.8. Definition. ([43]) Let $\mathrm{F}(X)$ be a linear space over $\mathbb{R}$. A fuzzy subset $N$ of $\mathrm{F}(X) \times \mathrm{F}(X) \times \mathbb{R}$ is called a 2-fuzzy 2-norm on $X$ (or fuzzy 2-norm on $\mathrm{F}(X)$ ) if (F2N1) for all $t \in \mathbb{R}$ with $t \leq 0 N\left(f_{1}, f_{2}, t\right)=0$; 
(F2N2) for all $t \in \mathbb{R}$ with $t>0, N\left(f_{1}, f_{2}, t\right)=1$, if and only if $f_{1}$ and $f_{2}$ are linearly dependent;

(F2N3) $N\left(f_{1}, f_{2}, t\right)$ is invariant under any permutation of $f_{1}, f_{2}$;

(F2N4) for all $t \in \mathbb{R}$, with $t>0, N\left(f_{1}, c f_{2}, t\right)=N\left(f_{1}, f_{2}, t /|c|\right)$ if $c \neq 0, c \in \mathbb{R}$;

(F2N5) for all $s, t \in \mathbb{R}, N\left(f_{1}, f_{2}+f_{3}, s+t\right) \geq \min \left\{N\left(f_{1}, f_{2}, s\right), N\left(f_{1}, f_{3}, t\right)\right\}$;

(F2N6) $N\left(f_{1}, f_{2},.\right):(0, \infty) \rightarrow[0,1]$ is continuous,

(F2N7) $\lim _{t \rightarrow \infty} N\left(f_{1}, f_{2}, t\right)=1$.

Then $(\mathrm{F}(X), N)$ is a fuzzy 2-normed linear space or $(X, N)$ is a 2-fuzzy 2-normed linear space.

2.9. Lemma. ([43, Theorem 3.2]) Let $(\mathrm{F}(X), N)$ be a fuzzy 2-normed linear space. Assume that

(F2N8) $N\left(f_{1}, f_{2}, t\right)>0$ for all $t>0$ implies $f_{1}$ and $f_{2}$ are linearly dependent, define

$$
\left\|f_{1}, f_{2}\right\|_{\alpha}=\inf \left\{t: N\left(f_{1}, f_{2}, t\right) \geq \alpha, \alpha \in(0,1)\right\} .
$$

Then $\left\{\|., .\|_{\alpha}: \alpha \in[0,1]\right\}$ is an ascending family of 2-norms on $\mathrm{F}(X)$. These 2-norms are called $\alpha$-2-norms on $\mathrm{F}(X)$ corresponding to the fuzzy 2-norms.

\section{J-convergence in 2-fuzzy 2-normed spaces}

In this section we introduce the notion of J-convergence and $\mathfrak{J}^{*}$-convergence of sequences in a 2-fuzzy 2-normed space $X$, i. e. in a fuzzy 2-normed space $(\mathrm{F}(X), N)$, and present some basic results.

3.1. Definition. Let $(\mathrm{F}(X), N)$ be fuzzy 2 -normed linear space. A sequence $\left\{f_{k}\right\}$ in $\mathrm{F}(X)$ is said to be $\mathcal{J}$-convergent to $f$ in $\mathrm{F}(X)$ with respect to the $\alpha$-2-norms on $\mathrm{F}(X)$ if for each $\epsilon>0, \alpha \in[0,1]$ and each $g \in \mathrm{F}(X)$, the set $A(\epsilon)=\{k \in \mathbb{N}$ : $\left.\left\|f_{k}-f, g\right\|_{\alpha} \geq \epsilon\right\}$ belongs to $\mathcal{J}$. In this case we write $f_{k} \stackrel{\mathcal{J}}{\rightarrow} f$. The element $f$ is called the J-limit of $\left\{f_{k}\right\}$ in $\mathrm{F}(X)$.

The usual interpretation of the above definition is the following:

$$
f_{k} \stackrel{I}{\rightarrow} f \Longleftrightarrow I-\lim _{k \rightarrow \infty}\left\|f_{k}-f, g\right\|_{\alpha}=0 \text {, for all } g \in \mathrm{F}(X) \text { and } \alpha \in[0,1] .
$$

3.2. Lemma. Let $(\mathrm{F}(X), N)$ be fuzzy 2-normed linear space and $\mathcal{J}$ be an admissible ideal of $\mathbb{N}$. If a sequence $\left\{f_{k}\right\}$ in $\mathrm{F}(X)$ is J-convergent with respect to the $\alpha$-2-norm on $\mathrm{F}(X)$, then $\mathcal{J}$-limit is unique.

Proof. Suppose that $f_{k} \stackrel{\mathcal{J}}{\rightarrow} f$ and $f_{k} \stackrel{\mathcal{J}}{\rightarrow} g$ and $f \neq g$. Since $\|.,\|_{\alpha}$ is an $\alpha$-2-norm, we get for each $h \in \mathrm{F}(X)$,

$$
\|f-g, h\|_{\alpha}=\left\|f_{k}-f_{k}+f-g, h\right\|_{\alpha} \leq\left\|f_{k}-f, h\right\|_{\alpha}+\left\|f_{k}-g, h\right\|_{\alpha}, \text { for all } k \in \mathbb{N} .
$$

Put

$$
\begin{aligned}
A(\varepsilon) & =\left\{k \in \mathbb{N}:\|f-g, h\|_{\alpha} \geq \varepsilon\right\}, \\
B\left(\frac{\varepsilon}{2}\right) & =\left\{k \in \mathbb{N}:\left\|f_{k}-f, h\right\|_{\alpha} \geq \frac{\varepsilon}{2}\right\}, \\
C\left(\frac{\varepsilon}{2}\right) & =\left\{k \in \mathbb{N}:\left\|f_{k}-g, h\right\|_{\alpha} \geq \frac{\varepsilon}{2}\right\} .
\end{aligned}
$$


By assumption, we get that $B\left(\frac{\varepsilon}{2}\right)$ and $C\left(\frac{\varepsilon}{2}\right)$ belong to J , so we have $B\left(\frac{\varepsilon}{2}\right) \cup C\left(\frac{\varepsilon}{2}\right) \in \mathcal{J}$. From (3.1), it follows that $A(\varepsilon) \subseteq B\left(\frac{\varepsilon}{2}\right) \cup C\left(\frac{\varepsilon}{2}\right)$. This implies that $A(\varepsilon) \in \mathcal{J}$, which achieves the proof.

3.3. Theorem. Let $(\mathrm{F}(X), N)$ be a fuzzy 2-normed linear space and $\mathcal{J}$ be an admissible ideal of $\mathbb{N}$. Let $\left\{f_{k}\right\}$ and $\left\{h_{k}\right\}$ be two sequences in $\mathrm{F}(X)$ such that $f_{k} \stackrel{\mathcal{J}}{\rightarrow} f$ and $h_{k} \stackrel{\mathcal{J}}{\rightarrow} h$, where $f, h \in \mathrm{F}(X)$. Then

(i) $f_{k}+h_{k} \stackrel{\mathcal{J}}{\rightarrow} f+h$;

(ii) $f_{k} h_{k} \stackrel{\mathcal{J}}{\rightarrow} f h$;

(iii) $c f_{k} \stackrel{\mathcal{J}}{\rightarrow} c f$ for $c \in \mathbb{R}$.

Proof. (i) Suppose that $f_{k} \stackrel{\mathcal{J}}{\rightarrow} f$ and $h_{k} \stackrel{\mathcal{J}}{\rightarrow} h$. Since $\|., .\|_{\alpha}$ is an $\alpha$-2-norm, we get for each $\varepsilon>0, g \in \mathrm{F}(X)$ and $\alpha \in[0,1]$,

$$
\left\|\left(f_{k}+h_{k}\right)-(f+h), g\right\|_{\alpha} \leq\left\|f_{k}-f, g\right\|_{\alpha}+\left\|h_{k}-h, g\right\|_{\alpha} \text {, for all } k \in \mathbb{N} .
$$

Put

$$
\begin{aligned}
A(\varepsilon) & =\left\{k \in \mathbb{N}:\left\|\left(f_{k}+h_{k}\right)-(f+h), g\right\|_{\alpha} \geq \varepsilon\right\}, \\
B\left(\frac{\varepsilon}{2}\right) & =\left\{k \in \mathbb{N}:\left\|f_{k}-f, g\right\|_{\alpha} \geq \frac{\varepsilon}{2}\right\}, \\
C\left(\frac{\varepsilon}{2}\right) & =\left\{k \in \mathbb{N}:\left\|h_{k}-h, g\right\|_{\alpha} \geq \frac{\varepsilon}{2}\right\} .
\end{aligned}
$$

By assumption, $B\left(\frac{\varepsilon}{2}\right)$ and $C\left(\frac{\varepsilon}{2}\right)$ belong to J , and thus $B\left(\frac{\varepsilon}{2}\right) \cup C\left(\frac{\varepsilon}{2}\right) \in \mathcal{J}$. From (3.2), it follows that $A(\varepsilon) \subseteq B\left(\frac{\varepsilon}{2}\right) \cup C\left(\frac{\varepsilon}{2}\right)$. This implies that $A(\varepsilon) \in \mathcal{J}$.

(ii) Since $f_{k} \stackrel{\mathcal{J}}{\rightarrow} f$, we have

$$
A(1)=\left\{k \in \mathbb{N}:\left\|f_{k}-f, g\right\|_{\alpha}<1\right\} \in \mathcal{F}(\mathcal{J}) .
$$

Now being $\alpha$-2-norm, we get

$$
\left\|f_{k} h_{k}-f h, g\right\|_{\alpha} \leq\left\|f_{k}, g\right\|_{\alpha}\left\|h_{k}-h, g\right\|_{\alpha}+\|h, g\|_{\alpha}\left\|f_{k}-f, g\right\|_{\alpha} .
$$

For $k \in A(1)$, we have $\left\|f_{k}, g\right\|_{\alpha} \leq\|f\|_{\alpha}+1$ and it follows that

$$
\left\|f_{k} h_{k}-f h, g\right\|_{\alpha} \leq\left(\|f\|_{\alpha}+1\right)\left\|h_{k}-h, g\right\|_{\alpha}+\|h, g\|_{\alpha}\left\|f_{k}-f, g\right\|_{\alpha} .
$$

Let $\varepsilon>0$ and $g \in \mathrm{F}(X)$ be given. Choose $\eta>0$ such that

$$
0<2 \eta<\frac{\varepsilon}{\|f\|_{\alpha}+\|h\|_{\alpha}+1} .
$$

Since $f_{k} \stackrel{\mathcal{J}}{\rightarrow} f$ and $h_{k} \stackrel{\mathcal{J}}{\rightarrow} h$, the sets

$$
B(\eta)=\left\{k \in \mathbb{N}:\left\|f_{k}-f, g\right\|_{\alpha}<\eta\right\} \text { and } C(\eta)=\left\{k \in \mathbb{N}:\left\|h_{k}-h, g\right\|_{\alpha}<\eta\right\} .
$$

belong to $\mathcal{F}(\mathcal{J})$. Thus we have $B(\eta), C(\eta) \in \mathcal{F}(\mathcal{J})$.

Obviously, $A(1) \cap B(\eta) \cap C(\eta) \in \mathcal{F}(\mathcal{J})$ and for each $k \in A(1) \cap B(\eta) \cap C(\eta)$, we have from (3.3) and (3.4),

$$
\left\|f_{k} h_{k}-f h, g\right\|_{\alpha}<\varepsilon \text {. }
$$

This implies that $\left\{k \in \mathbb{N}:\left\|f_{k} h_{k}-f h, g\right\|_{\alpha} \geq \varepsilon\right\} \in \mathcal{J}$, i.e., $f_{k} g_{k} \stackrel{\mathcal{J}}{\rightarrow} f h$. 
(iii) It is trivial for $c=0$. Now let $c \neq 0, \varepsilon>0, \alpha \in[0,1]$ and $g \in \mathrm{F}(X)$. Since $\|., .\|_{\alpha}$ is an $\alpha$-2-norm, we get $\|c f, g\|_{\alpha}=|c|\|f, g\|$. Since $f_{k} \stackrel{\mathcal{J}}{\rightarrow} f$, therefore the set

$$
A=\left\{k \in \mathbb{N}:\left\|f_{k}-f, g\right\|_{\alpha} \geq \varepsilon\right\}
$$

belongs J. Let $B(\varepsilon)=\left\{k \in \mathbb{N}:\left\|c f_{k}-c f, g\right\|_{\alpha} \geq \varepsilon\right\}$. We need to show that $B(\varepsilon)$ is contained in $A\left(\varepsilon_{1}\right)$, for some $\varepsilon_{1}>0$. Let $m \in B(\varepsilon)$; then $\varepsilon \leq\left\|c f_{m}-c f, g\right\|_{\alpha}=$ $|c|\left\|f_{m}-f, g\right\|_{\alpha}$. This implies that $\left\|f_{m}-f, g\right\|_{\alpha} \geq \frac{\varepsilon}{|c|}=\varepsilon_{1}$. Therefore, $m \in A\left(\varepsilon_{1}\right)$. Then we have $B(\varepsilon) \subset A\left(\varepsilon_{1}\right)$. By the definition of $\mathcal{J}$, we get $B(\varepsilon) \in \mathcal{J}$.

The theorem is proved.

3.4. Theorem. Let $\mathcal{J}$ be an admissible ideal with the property (AP). Let $(\mathrm{F}(X), N)$ be a fuzzy 2-normed space and $\left\{f_{k}\right\}$ be a sequence in $\mathrm{F}(X)$. Then $\left\{f_{k}\right\}$ is an Jconvergent sequence in $\mathrm{F}(X)$ if and only if there is a sequence $\left\{h_{k}\right\}$ converging to $f$ and such that $\left\{k \in \mathbb{N}: f_{k} \neq h_{k}\right\} \in \mathcal{J}$.

Proof. Suppose $f_{k} \stackrel{\mathcal{J}}{\rightarrow} f$. For each $k \in \mathbb{N}$ and each $g \in \mathrm{F}(X)$, let

$$
A_{n}=\left\{k \in \mathbb{N}:\left\|f_{k}-f, g\right\|_{\alpha}<\frac{1}{n}\right\},
$$

Then $A_{n} \in \mathcal{F}(\mathcal{J})$ for each $n \in \mathbb{N}$.

Since $\mathcal{J}$ is admissible ideal with the property (AP), by Lemma 1.1, there is $A \subset \mathbb{N}$ such that $A \in \mathcal{F}(\mathcal{J})$ and the set $A \backslash A_{n}$ is finite for each $n$. Observe that $f_{k} \rightarrow_{(A)} f$, i.e., for each $\varepsilon>0$, there exists $n_{0}=n_{0}(\varepsilon) \in \mathbb{N}$ such that $k \geq n_{0}$ and $k \in A$ imply $\left\|f_{k}-f, g\right\|_{\alpha}<\varepsilon$.

Define a sequence $\left\{h_{k}\right\}$ in $\mathrm{F}(X)$ as

$$
h_{k}= \begin{cases}f_{k}, & \text { for } k \in A \\ f, & \text { for } k \in \mathbb{N} \backslash A .\end{cases}
$$

The sequence $\left\{h_{k}\right\}$ is convergent to $f$ with respect to the $\alpha$-2-norm on $\mathrm{F}(X)$. Thus we have $\left\{k \in \mathbb{N}: f_{k} \neq h_{k}\right\} \in \mathcal{J}$.

Conversely, suppose that $\left\{k \in \mathbb{N}: f_{k} \neq h_{k}\right\} \in \mathcal{J}$ and $h_{k} \rightarrow f$. Let $\varepsilon>0$ and $g \in \mathrm{F}(X)$ be given. Then for each $n$, we can write

$$
\left\{k \leq n:\left\|f_{k}-f, g\right\|_{\alpha} \geq \varepsilon\right\} \subseteq\left\{k \leq n: f_{k} \neq h_{k}\right\} \cup\left\{k \leq n:\left\|h_{k}-f, g\right\|_{\alpha}>\varepsilon\right\} .
$$

Since the first set on the right side of (3.5) belongs to $\mathcal{J}$ and the second set is contained in a finite subset of $\mathbb{N}$, it belongs to $\mathcal{J}$. This implies that $\{k \in \mathbb{N}$ : $\left.\left\|f_{k}-f, g\right\|_{\alpha} \geq \varepsilon\right\}$ belongs to $\mathcal{J}$. This achieves the proof.

Now we prove a decomposition theorem for J-convergent sequences.

3.5. Theorem. Let $\left\{f_{k}\right\}$ be a sequence in a fuzzy 2-normed space $(\mathrm{F}(X), N)$ and let $\mathcal{J}$ be an admissible ideal with the property (AP). Then the following assertions are equivalent:

(i) $f_{k} \stackrel{\mathcal{J}}{\rightarrow} f$;

(ii) There exist $\left\{h_{k}\right\}$ and $\left\{q_{k}\right\}$ in $\mathrm{F}(X)$ such that $f_{k}=h_{k}+q_{k}, h_{k} \rightarrow h$, and $\operatorname{supp}\left(q_{k}\right)=\left\{k \in \mathbb{N}: q_{k} \neq \theta\right\} \in \mathcal{J}$, where $\theta$ is the zero element of the linear space $\mathrm{F}(X)$. 
Proof. (i) $\Longrightarrow$ (ii) Suppose $f_{k} \stackrel{\mathcal{J}}{\rightarrow} f$. Then, by Lemma 1.2, we there is a set $H \in$ $\mathcal{F}(\mathcal{J}), H=\left\{k_{m}: k_{1}<k_{2}<\cdots\right\} \subset \mathbb{N}$ such that $f_{k_{m}} \rightarrow f$.

Define the sequence $\left\{h_{k}\right\}$ in $\mathrm{F}(X)$ as

$$
h_{k}= \begin{cases}f_{k}, & \text { for } k \in H \\ f, & \text { for } k \in \mathbb{N} \backslash H .\end{cases}
$$

It is clear that $h_{k} \rightarrow f$. Further, we set $q_{k}=f_{k}-h_{k}$, for each $k \in \mathbb{N}$. Since $\left\{k \in \mathbb{N}: f_{k} \neq h_{k}\right\} \in \mathbb{N} \backslash H \in \mathcal{J}$, we have $\left\{k \in \mathbb{N}: q_{k} \neq \theta\right\} \in \mathcal{J}$. It follows that $\operatorname{supp}\left(q_{k}\right) \in \mathcal{J}$ and by (3.6), we get $f_{k}=h_{k}+q_{k}$.

(ii) $\Longrightarrow$ (i) Suppose that there exist two sequences $\left\{h_{k}\right\}$ and $\left\{q_{k}\right\}$ in $\mathrm{F}(X)$ such that $f_{k}=h_{k}+q_{k} ; h_{k} \rightarrow f$ and $\operatorname{supp}\left(q_{k}\right) \in \mathcal{J}$. We prove that $f_{k} \stackrel{\mathcal{J}}{\rightarrow} f$. Let $H=\left\{k_{m}\right\}$ be a subset of $\mathbb{N}$ such that $H=\left\{k \in \mathbb{N}: q_{k}=\theta\right\}$. Since $\operatorname{supp}\left(q_{k}\right)=\left\{m \in \mathbb{N}: q_{m} \neq\right.$ $\theta\} \in \mathcal{J}$, we have $H \in \mathcal{F}(\mathcal{J})$, therefore $f_{k}=h_{k}$, if $k \in H$. Thus, we conclude that there exists a set $H=\left\{k_{m}: k_{1}<k_{2}<\cdots\right\} \subset \mathbb{N}, H \in \mathcal{F}(\mathcal{J})$, such that $f_{k_{m}} \rightarrow f$. By Lemma 1.2, it follows that $f_{k} \stackrel{\mathcal{J}}{\rightarrow} f$.

3.6. Definition. Let $(\mathrm{F}(X), N)$ be a fuzzy 2-normed space. We say that a sequence $\left\{f_{k}\right\}$ in $\mathrm{F}(X)$ is $\mathcal{J}^{*}$-convergent to $f \in \mathrm{F}(X)$ with respect to the $\alpha$-2-norm on $\mathrm{F}(X)$ if there exists a subset

$$
K=\left\{k_{m}: k_{1}<k_{2}<\cdots\right\} \subset \mathbb{N}
$$

such that $K \in \mathcal{J}$ and $\lim _{m \rightarrow \infty}\left\|f_{k_{m}}-f, g\right\|_{\alpha}=0$ for each $g \in \mathrm{F}(X)$.

In this case we write $f_{k} \stackrel{\mathcal{J}^{*}}{\rightarrow} f$.

3.7. Theorem. Let $(\mathrm{F}(X), N)$ be a fuzzy 2-normed space and $\mathcal{J}$ be an admissible ideal. If $f_{k} \stackrel{\mathcal{J}^{*}}{\rightarrow} f$, then $f_{k} \stackrel{\mathcal{J}}{\rightarrow} f$.

Proof. Suppose that $f_{k} \stackrel{\mathcal{J}^{*}}{\rightarrow} f$. Then, by definition, there exists

$$
K=\left\{k_{m} \in \mathbb{N}: k_{1}<k_{2}<\cdots\right\} \in \mathcal{F}(\mathcal{J})
$$

such that

$$
\lim _{m \rightarrow \infty}\left\|f_{k_{m}}-f, g\right\|_{\alpha}=0 \text {, for all } g \in \mathrm{F}(X) .
$$

Let $\varepsilon>0$ and $g \in \mathrm{F}(X)$ be given. By (3.7, there exists an integer $n_{0} \in \mathbb{N}$ such that $\left\|f_{k_{m}}-f, g\right\|_{\alpha}<\varepsilon$ for every $k_{m} \in K, k_{m} \geq n_{0}$.

Let $A=\left\{k_{1}, k_{2}, \cdots, k_{n_{0}}\right\}$. Since $K \in \mathcal{F}(\mathcal{J})$, there exists a set $B \in \mathcal{J}$ such that $K=\mathbb{N} \backslash B$. It is clear

$$
A_{1}(\varepsilon)=\left\{k \in \mathbb{N}:\left\|f_{k}-f, g\right\|_{\alpha} \geq \varepsilon\right\} \subseteq A \cup B .
$$

As $\mathcal{J}$ is admissible ideal, $A \in \mathcal{J}$. This implies that $A \cup B \in \mathcal{J}$ and so $A_{1}(\varepsilon) \in \mathcal{J}$. Thus we have $f_{k} \stackrel{\mathcal{J}}{\rightarrow} f$. This completes the proof.

3.8. Theorem. Let $\mathcal{J}$ be an admissible ideal with the property $(\mathrm{AP})$ and $(\mathrm{F}(X), N)$ be a fuzzy 2-normed space and $\left\{f_{k}\right\}$ be a sequence in $\mathrm{F}(X)$. Then $f_{k} \stackrel{\mathcal{J}}{\rightarrow} f$ if and only if $f_{k} \stackrel{\mathcal{J}^{*}}{\longrightarrow} f$. 
Proof. If $f_{k} \stackrel{\mathcal{J}^{*}}{\rightarrow} f$, then $f_{k} \stackrel{\mathcal{J}}{\rightarrow} f$ by Theorem 3.7.

Conversely, let $f_{k} \stackrel{\mathcal{J}}{\rightarrow} f$. Then by definition, for each $\varepsilon>0$ and each $g \in \mathrm{F}(X)$, there exists an integer $n=n(\varepsilon)$ such that

$$
B(\varepsilon)=\left\{k \in\left\|f_{k}\right\|_{\alpha} \geq \varepsilon\right\} \in \mathcal{J} .
$$

For $m \in \mathbb{N}$, we define the set $P_{m}$ as follows:

$$
\begin{aligned}
& P_{1}=\left\{k \in \mathbb{N}:\left\|f_{k}-f, g\right\|_{\alpha} \geq 1\right\}, \\
& P_{m}=\left\{k \in \mathbb{N}: \frac{1}{m} \leq\left\|f_{k}-f, g\right\|_{\alpha}<\frac{1}{m-1}\right\}, \text { for } m \geq 2 \text { in } \mathbb{N} .
\end{aligned}
$$

It is clear that $\left\{P_{1}, P_{2}, \cdots\right\}$ is a countable family of mutually disjoint sets belonging to $\mathcal{J}$. Then by the property (AP) of $\mathcal{J}$, there is a countable family of sets $\left\{Q_{1}, Q_{2}, \cdots\right\}$ in $\mathcal{J}$ such that $P_{j} \Delta Q_{j}$ is a finite set for each $j \in \mathbb{N}$ and $Q=$ $\bigcup_{j=1}^{\infty} Q_{j} \in \mathcal{J}$. Since $Q \in \mathcal{J}$, we have $B=\mathbb{N} \backslash Q \in \mathcal{F}(\mathcal{J})$. To prove the result it is sufficient to show that $f_{k} \rightarrow_{(B)} f$. Let $\xi>0$ be given. Choose an integer $p$ such that $\xi>\frac{1}{p+1}$. Thus, we have

$$
\left\{k \in \mathbb{N}:\left\|f_{k}-f, g\right\|_{\alpha} \geq \xi\right\} \subset\left\{k \in \mathbb{N}:\left\|f_{k}-f, g\right\|_{\alpha} \geq \frac{1}{p+1}\right\}=\bigcup_{m=1}^{p+1} P_{m} .
$$

Since $P_{m} \Delta Q_{m}$ is a finite set for each $m=1, \cdots, p+1$, therefore there exists $k_{0} \in \mathbb{N}$ such that

$$
\left(\bigcup_{m=1}^{p+1} Q_{m}\right) \cap\left\{k \in \mathbb{N}: k \geq k_{0}\right\}=\bigcup_{m=1}^{p+1} P_{m} \cap\left\{k \in \mathbb{N}: k \geq k_{0}\right\} .
$$

If $k \geq k_{0}$ and $k \in Q$, then $k \notin \bigcup_{m=1}^{p+1} Q_{m}$ and so $k \notin \bigcup_{m=1}^{p+1} P_{m}$. Thus for every $k \geq k_{0}$ and $k \in B$, from (3.8), we get $\left\|f_{k}-f, g\right\|_{\alpha}<\xi$. This shows $f_{k} \rightarrow_{(B)} f$ which completes the proof.

The proof of the following theorem follows from the decomposition theorem (Theorem 3.5).

3.9. Theorem. Let $\left\{f_{k}\right\}$ be a sequence in a fuzzy 2-normed space $(\mathrm{F}(X), X)$ and $\mathcal{J}$ be an admissible ideal. If there exist two sequences $\left\{h_{k}\right\}$ and $\left\{q_{k}\right\}$ in $\mathrm{F}(X)$ such that $f_{k}=h_{k}+q_{k} ; h_{k} \rightarrow f$ and $\operatorname{supp}\left(q_{k}\right)=\left\{k \in \mathbb{N}: q_{k} \neq \theta\right\} \in \mathcal{J}$, then $f_{k} \stackrel{J^{*}}{\rightarrow} f$.

\section{J-Cauchy and $J^{*}$-Cauchy sequences in 2-fuzzy 2-normed spaces}

In this section we study the concepts of J-Cauchy and $\mathcal{J}^{*}$-Cauchy sequences in fuzzy 2-normed spaces $(\mathrm{F}(X), N)$. Also, we will study the relations between these concepts. For statistical Cauchy sequences and J-Cauchy sequences see [15, 16, 35].

4.1. Definition. Let $(\mathrm{F}(X), N)$ be a fuzzy 2-normed space and $\mathcal{J}$ be an admissible ideal of $\mathbb{N}$. A sequence $\left\{f_{k}\right\}$ in $\mathrm{F}(X)$ is said to be J-Cauchy with respect to the $\alpha$-2-norm on $\mathrm{F}(X)$ if for each $\varepsilon>0$ there exist a positive integer $n=n(\varepsilon)$ and $g, h \in \mathrm{F}(X)$ which are linearly independent such that $\left\{k \in \mathbb{N}:\left\|f_{k}-f_{n}, g\right\|_{\alpha} \geq \varepsilon\right\}$ and $\left\{k \in \mathbb{N}:\left\|f_{k}-f_{n}, h\right\|_{\alpha} \geq \varepsilon\right\}$ belong to J. 
4.2. Definition. Let $(\mathrm{F}(X), N)$ be a fuzzy 2-normed space and $\mathcal{J}$ be an admissible ideal of $\mathbb{N}$. A sequence $\left\{f_{k}\right\}$ in $\mathbf{F}(X)$ is said to be $\mathfrak{J}^{*}$-Cauchy with respect to the $\alpha$-2-norm on $\mathrm{F}(X)$ if there exists a set

$$
K=\left\{k_{m}: k_{1}<k_{2}<\cdots\right\} \subset \mathbb{N}
$$

such that $K \in \mathcal{F}(\mathcal{J})$ and $\left\{f_{k_{m}}\right\}$ is an ordinary Cauchy sequence in $\mathrm{F}(X)$.

The next theorem gives that each $\mathcal{J}^{*}$-Cauchy sequence is J-Cauchy sequence.

4.3. Theorem. Let $\mathcal{J}$ be an admissible ideal and $(\mathrm{F}(X), N)$ be a fuzzy 2-normed space. Then every J-convergent sequence is an J-Cauchy sequence.

Proof. Let $f_{k} \stackrel{\mathcal{J}}{\rightarrow} f$. Then for each $\varepsilon>0$ and each $\phi \in \mathrm{F}(X)$, we have

$$
A(\varepsilon)=\left\{k \in \mathbb{N}:\left\|f_{k}-f, \phi\right\|_{\alpha} \geq \varepsilon\right\} \in \mathcal{J} .
$$

Since $\mathcal{J}$ is an admissible ideal, there exists an $k_{0} \in \mathbb{N}$ such that $k_{0} \notin A(\varepsilon)$.

Let $B(\varepsilon)=\left\{k \in \mathbb{N}:\left\|f_{k}-f_{k_{0}}, \phi\right\|_{\alpha} \geq 2 \varepsilon\right\}$. Since $\|., .\|_{\alpha}$ is an $\alpha$-2-norm, we get

$$
\left\|f_{k}-f_{k_{0}}, \phi\right\|_{\alpha} \leq\left\|f_{k}-f, \phi\right\|_{\alpha}+\left\|f_{k_{0}}-f, \phi\right\|_{\alpha} .
$$

We observe that if $k \in B(\varepsilon)$, then $\left\|f_{k}-f, \phi\right\|_{\alpha}+\left\|f_{k_{0}}-f, \phi\right\|_{\alpha} \geq 2 \varepsilon$.

On the other hand, since $k_{0} \notin A(\varepsilon)$, we have

$$
\left\|f_{k_{0}}-f, \phi\right\|_{\alpha}<\varepsilon \text {. }
$$

So we conclude that $\left\|f_{k}-f, \phi\right\|_{\alpha} \geq \varepsilon$, hence $k \in A(\varepsilon)$.

This implies that $B(\varepsilon) \subset A(\varepsilon)$, for each $\varepsilon>0$. This gives $B(\varepsilon) \in \mathcal{J}$. Since $\phi \in \mathrm{F}(X)$ was arbitrary we can take $g, h \in \mathrm{F}(X)$ which are linearly independent, such that the sets $\left\{k \in \mathbb{N}:\left\|f_{k}-f_{k_{0}}, g\right\|_{\alpha} \geq 2 \varepsilon\right\}$ and $\left\{k \in \mathbb{N}:\left\|f_{k}-f_{k_{0}}, h\right\|_{\alpha} \geq 2 \varepsilon\right\}$ belong to J, i.e., $\left\{f_{k}\right\}$ is an J-Cauchy sequence.

4.4. Theorem. Let $(\mathrm{F}(X)), N)$ be a fuzzy 2-normed space and $\mathcal{J}$ be an admissible ideal of $\mathbb{N}$. If $\left\{f_{k}\right\}$ is $\mathcal{J}^{*}$-Cauchy sequence, then it is an J-Cauchy sequence.

Proof. Let $\left\{f_{k}\right\}$ be an $\mathcal{J}^{*}$-Cauchy sequence. Then for $\varepsilon>0$ and each $\phi \in \mathrm{F}(X)$, there are

$$
K=\left\{k_{m}: k_{1}<k_{2}<\cdots\right\} \in \mathcal{F}(\mathcal{J})
$$

and a number $n_{0} \in \mathbb{N}$ such that

$$
\left\|f_{k_{m}}-f_{k_{p}}, \phi\right\|_{\alpha}<\varepsilon
$$

for every $m, p \geq n_{0}$. Now, fix $p=k_{n_{0}+1}$. Then for every $\varepsilon>0$ and each $\phi \in \mathrm{F}(X)$, we have

$$
\left\|f_{k_{m}}-f_{p}, \phi\right\|_{\alpha}<\varepsilon \text { for every } m \geq n_{0} .
$$

Let $H=\mathbb{N} \backslash K$. It is obvious that $H \in \mathcal{J}$ and

$$
A(\varepsilon)=\left\{k \in \mathbb{N}:\left\|f_{k_{m}}-f_{p}, \phi\right\|_{\alpha} \geq \varepsilon\right\} \subset H \cup\left\{k_{1}<k_{2}<\cdots<k_{n_{0}}\right\} \in \mathcal{J} .
$$

Therefore, for every $\varepsilon>0$, we can find $p \in \mathbb{N}$ and $g, h \in \mathrm{F}(X)$ such that the sets

$$
\left\{k \in \mathbb{N}:\left\|f_{k_{m}}-f_{p}, g\right\|_{\alpha} \geq \varepsilon\right\} \text { and }\left\{k \in \mathbb{N}:\left\|f_{k_{m}}-f_{p}, h\right\|_{\alpha} \geq \varepsilon\right\}
$$

belong to J, i.e., $\left\{f_{k}\right\}$ is an J-Cauchy sequence. 
Now we prove that $\mathcal{J}^{*}$-convergence implies J-Cauchy condition in a fuzzy 2normed space $(\mathrm{F}(X), N)$.

4.5. Theorem. Let $(\mathrm{F}(X), N)$ be a fuzzy 2-normed space and $\mathcal{J}$ be an admissible ideal of $\mathbb{N}$. If $\left\{f_{k}\right\}$ is $\mathcal{J}^{*}$-convergent, then it is an J-Cauchy sequence.

Proof. By assumption there exists a set $K=\left\{k_{m}: k_{1}<k_{2}<\cdots\right\}$ such that $K \in \mathcal{F}(\mathcal{J})$ and $\lim _{m}\left\|f_{k_{m}}-f, \phi\right\|_{\alpha}=0$ for each $\phi \in \mathrm{F}(X)$, i.e., there exists $n_{0} \in \mathbb{N}$ such that $\left\|f_{k_{m}}-f, \phi\right\|_{\alpha}<\varepsilon$ for every $\varepsilon>0$, each $\phi \in \mathrm{F}(X)$ and $m>n_{0}$. Since $\|., .\|_{\alpha}$ is an $\alpha$-2-norm, we have

$$
\left\|f_{k_{m}}-f_{k_{p}}, \phi\right\|_{\alpha} \leq\left\|f_{k_{m}}-f, \phi\right\|_{\alpha}+\left\|f_{k_{p}}-f, \phi\right\|_{\alpha}<2 \varepsilon
$$

for every $\varepsilon>0$, each $\phi \in \mathrm{F}(X)$ and $m, p>n_{0}$, we have $\left\|f_{k_{m}}-f_{k_{p}}, \phi\right\|_{\alpha}<2 \varepsilon$ for every $m, p>n_{0}$ and each $\phi \in \mathrm{F}(X)$, i.e., $\left\{f_{k}\right\}$ is an $\mathcal{J}^{*}$-Cauchy sequence in $\mathrm{F}(X)$. Therefore, it follows from Theorem 4.4 that $\left\{f_{k}\right\}$ is an J-Cauchy sequence.

4.6. Theorem. Let $\mathcal{J}$ be an admissible ideal. Let $\left\{f_{k}\right\}$ be a sequence in a fuzzy 2-normed space $(\mathrm{F}(X), N)$ and denote $A(\varepsilon)=\left\{k \in \mathbb{N}:\left\|f_{k}-f_{n}, g\right\|_{\alpha} \geq \varepsilon\right\}$, where $n \in \mathbb{N}$ and $g \in \mathrm{F}(X)$. If $\left\{f_{k}\right\}$ is an J-Cauchy sequence, then for every $\varepsilon>0$ and $g \in \mathrm{F}(X)$ there exists $B \in \mathcal{J}$ such that $\left\|f_{l}-f_{k}, g\right\|_{\alpha}<\varepsilon$, for all $k, l \notin B$.

Proof. Let $\varepsilon>0$ and $g \in \mathrm{F}(X)$ be given. Set $B=A_{n}(\varepsilon / 2)$, where $n \in \mathbb{N}$. Since $\left\{f_{k}\right\}$ is an J-Cauchy sequence, we have $B \in \mathcal{J}$ and for all $l, k \notin B$, we get

$$
\left\|f_{k}-f_{n}, g\right\|_{\alpha}<\frac{\varepsilon}{2} \text { and }\left\|f_{l}-f_{n}, g\right\|_{\alpha}<\frac{\varepsilon}{2} .
$$

Because $\|.,$.$\| is an \alpha$-2-norm, by the triangle inequality we have $\left\|f_{k}-f_{l}, g\right\|_{\alpha}<\varepsilon$, for all $l, k \notin B$.

\section{J-limit points and J-cluster points}

In this section we introduce the notion of J-limit point and J-cluster point of real sequences in 2-fuzzy 2-normed linear spaces.

5.1. Definition. Let $\left\{f_{k}\right\}$ be a sequence in a fuzzy 2-normed space $(\mathrm{F}(X), N)$. An element $\psi \in \mathrm{F}(X)$ is said to be an J-limit point of $\left\{f_{k}\right\}$ provided there is a set $K=\left\{k_{1}<k_{2}<\cdots<k_{m}<\cdots\right\} \subset \mathbb{N}$ such that $K \notin \mathcal{J}$ and $\lim _{m}\left\|f_{k_{m}}-\psi, g\right\|_{\alpha}=$ 0 , for every $g \in \mathrm{F}(X)$.

5.2. Definition. Let $\left\{f_{k}\right\}$ be a sequence in a fuzzy 2-normed space $(\mathrm{F}(X), N)$. An element $\phi \in \mathrm{F}(X)$ is said to be an J-cluster point of $\left\{f_{k}\right\}$ if for every $\varepsilon>0$ and each $g \in \mathrm{F}(X)$, the set $\left\{k \in \mathbb{N}:\left\|f_{k}-\phi, g\right\|_{\alpha}<\varepsilon\right\} \notin \mathcal{J}$.

We denote $L_{\mathrm{F}(X)}^{\mathcal{J}}\left(f_{k}\right)$ and $C_{\mathrm{F}(X)}^{\mathcal{J}}\left(f_{k}\right)$ the set of of all J-limit points and J-cluster points of a sequence $\left\{f_{k}\right\}$ in $(\mathrm{F}(X), N)$, respectively.

5.3. Theorem. Let $\mathcal{J}$ be an admissible ideal. Then for any $\left\{f_{k}\right\}$ in a fuzzy $2-$ normed space $(\mathrm{F}(X), N)$, we have

$$
L_{\mathrm{F}(X)}^{\mathcal{J}}\left(f_{k}\right) \subset C_{\mathrm{F}(X)}^{\mathcal{J}}\left(f_{k}\right) .
$$


Proof. Suppose that $\psi \in L_{\mathrm{F}(X)}^{\mathcal{J}}\left(f_{k}\right)$. Then there exists a set $K=\left\{k_{1}<k_{2}<\cdots<\right.$ $\left.k_{m}<\cdots\right\} \subset \mathbb{N}$ such that $K \notin \mathcal{J}$ and

$$
\lim _{m \rightarrow \infty}\left\|f_{k}-\psi, g\right\|_{\alpha}=0 \text { for each } g \in \mathrm{F}(X) \text {. }
$$

Let $\varepsilon>0$ and $g \in \mathrm{F}(X)$ be given. According to (5.1), there exists an integer $n_{0}=n_{0}(\varepsilon) \in \mathbb{N}$ such that for $k \geq n_{0}$, we have $\left\|f_{k}-\psi, g\right\|_{\alpha}<\varepsilon$. Thus we have

$$
K \backslash\left\{k_{1}, k_{2}, \cdots, k_{n_{0}}\right\} \subset\left\{k \in \mathbb{N}:\left\|f_{k}-\psi, g\right\|_{\alpha}<\varepsilon\right\} .
$$

This implies that $\left\{k \in \mathbb{N}:\left\|f_{k}-\psi, g\right\|_{\alpha}<\varepsilon\right\} \notin \mathcal{J}$. Therefore, $\psi \in C_{\mathrm{F}(X)}^{\mathcal{J}}\left(f_{k}\right)$.

5.4. Theorem. Let $\left\{f_{k}\right\}$ be a sequence in a fuzzy 2-normed space $(\mathrm{F}(X), N)$. If $f_{k} \stackrel{\mathcal{J}}{\rightarrow} f$, then

$$
L_{\mathrm{F}(X)}^{\mathcal{J}}\left(f_{k}\right)=C_{\mathrm{F}(X)}^{\mathcal{J}}\left(f_{k}\right)=\{f\} .
$$

Proof. Suppose that $f_{k} \stackrel{\mathcal{J}}{\rightarrow} f$. Then for each $\varepsilon>0$ and $g \in \mathrm{F}(X)$, we have

$$
\left\{k \in \mathbb{N}:\left\|f_{k}-f, g\right\|_{\alpha} \geq \varepsilon\right\} \in \mathcal{J} \text {, i.e. }\left\{k \in \mathbb{N}:\left\|f_{k}-f, g\right\|_{\alpha}<\varepsilon\right\} \notin \mathcal{J},
$$

which implies that $f \in C_{\mathrm{F}(X)}^{\mathcal{J}}\left(f_{k}\right)$.

We assume that there exists at least one $h \in C_{\mathrm{F}(X)}^{\mathcal{J}}\left(f_{k}\right)$ such that $h \neq f$. Then there exists $\varepsilon>0$ such that

$$
\left\{k \in \mathbb{N}:\left\|f_{k}-f, g\right\|_{\alpha} \geq \varepsilon\right\} \supset\left\{k \in \mathbb{N} ;\left\|f_{k}-h, g\right\|_{\alpha}<\varepsilon\right\} .
$$

But $\left\{k \in \mathbb{N}:\left\|f_{k}-f, g\right\|_{\alpha} \geq \varepsilon\right\} \in \mathcal{J}$ implies that $\left\{k \in \mathbb{N}:\left\|f_{k}-h, g\right\|_{\alpha}<\varepsilon\right\} \in \mathcal{J}$, which contradicts that $h \in C_{\mathrm{F}(X)}^{\mathcal{J}}\left(f_{k}\right)$. Thus we have $C_{\mathrm{F}(X)}^{\mathcal{J}}\left(f_{k}\right)=\{f\}$.

On the other hand, from $f_{k} \stackrel{\mathcal{J}}{\rightarrow} f$, by Theorem 3.4 and Definition 5.2, we have $f \in C_{\mathrm{F}(X)}^{\mathcal{J}}\left(f_{k}\right)$. By Theorem 5.3, we have $L_{\mathrm{F}(X)}^{\mathcal{J}}\left(f_{k}\right)=C_{\mathrm{F}(X)}^{\mathcal{J}}\left(f_{k}\right)=\{f\}$.

\section{References}

[1] S. Aytar, Statistical limit points of sequences of fuzzy numbers, Inform. Sci. 165 (2004), 129-138.

[2] S. Aytar, M. Mammadov, S. Pehlivan, Statistical limit inferior and limit superior for sequences of fuzzy numbers, Fuzzy Sets Syst. 157:7 (2006), 976-985.

[3] S. Aytar, S. Pehlivan, Statistical cluster and extreme limit points of sequences of fuzzy numbers, Inform. Sci. 177 (2007), 3290-3296.

[4] T. Bag, S.K. Samanta, Finite dimensional fuzzy normed linear spaces, J. Fuzzy Math. 11:3 (2003), 687-705

[5] T. Bag, S.K. Samanta, Fuzzy bounded linear operators, Fuzzy Sets Syst. 151:3 (2005), $513-547$.

[6] T. Bag, S.K. Samanta, A comparative study of fuzzy norms on a linear space, Fuzzy Sets Syst. 159 (2008), 670-684.

[7] M. Balcerzak, K. Dems, A. Komisarski, Statistical convergence and ideal convergence for sequences of functions, J. Math. Anal. Appl. 328 (2007), 715-729.

[8] A.I. Bernstein, A new kind of compactness for topological spaces, Fund. Math. 66 (1970), $185-193$.

[9] H. Çakalli, S. Ersan, Strongly lacunary ward continuity in 2-normed spaces, Sci. World J. 2014 (2014), Art. ID 479679, 5 pp.

[10] H. Çakalli, S. Ersan, New types of continuity in 2-normed spaces, Filomat 30:3 (2016), $525-532$.

[11] A. Caserta, G. Di Maio, Lj.D.R. Kočinac, Statistical convergence in function spaces, Abstr. Appl. Anal. 2011 (2011), Article ID 420419, 11 pages. 
[12] S.C. Cheng, J.N. Mordeson, Fuzzy linear operators and fuzzy normed linear spaces, Bull. Cal. Math. Soc 86 (1994), 429-436.

[13] P. Das, S.K. Ghosal, Some further results on J-Cauchy sequences and condition (AP), Comput. Math. Appl. 59 (2010), 2597-2600.

[14] P. Das, S. Pal, S.K. Ghosal, Further investigations of ideal summability in 2-normed spaces, Appl. Math. Letters 24 (2011), 39-43.

[15] P. Das, E. Savaş, On J-statistically pre-Cauchy sequences, Taiwanese J. Math. 18:1 (2014), $115-126$.

[16] K. Dems, On J-Cauchy sequences, Real Anal. Exch. 30:1 (2004-2005), 123-128.

[17] G. Di Maio, Lj.D.R. Kočinac, Statistical convergence in topology, Topology Appl. 156:1 (2008), 28-45.

[18] S. Ersan, H. Çakalli, Ward continuity in 2-normed spaces, Filomat 29:7 (2015), 1507-1513.

[19] S. Gähler, 2-metrishe Räume und ihr topologishe struktur, Math. Nachr. 26 (1963), 115-148.

[20] H. Fast, Sur la convergence statistique, Colloq. Math. 2 (1951), 241-244.

[21] C. Felbin, Finite dimensional fuzzy normed linear spaces, Fuzzy Sets Syst. 48 (1992), 239248.

[22] J.A. Fridy, On statistical convergence, Analysis 5 (1985), 301-313.

[23] B. Hazarika, On ideal convergent sequences in fuzzy normed linear spaces, Afrika Matematika 25:4 (2014), 987-999.

[24] B. Hazarika, E. Savaş, Some J-convergent lamda-summable difference sequence spaces of fuzzy real numbers defined by a sequence of Orlicz, Math. Comp. Modelling 54 (2011), $2986-2998$.

[25] O. Kaleva, S. Seikkala, On fuzzy metric spaces, Fuzzy Sets Syst. 12 (1984), 215-229.

[26] S. Karakuş, K. Demirci, O. Duman, Statistical convergence on intuitionistic fuzzy normed spaces, Chaos, Solitons and Fractals 35 (2008), 763-769.

[27] V. Karakaya, N.N. Şimşek, M. Ertürk, F. Gürsoy, Statistical A-convergence of sequences of functions in intuitionistic fuzzy normed spaces, Abstr. Appl. Anal. 2012 (2012).

[28] V. Karakaya, N.N. Şimşek, M. Ertürk, F. Gürsoy, On ideal convergence of sequences of functions in intuitionistic fuzzy normed spaces, Appl. Math. Inf. Sci. 8:5 (2014), 2307-2313.

[29] A.K. Katsaras, Fuzzy topological vector spaces, Fuzzy Sets Syst. 12 (1984), 143-154.

[30] P. Kostyrko, T. Šalát, W. Wilczyński, J-convergence, Real Anal. Exchange, 26:2 (2000-2001), 669-686.

[31] P. Kostyrko, M. Mačaj, T. Šalát, M. Sleziak, J-convergence and extremal J-limit points, Math. Slovaca 55 (2005), 443-464.

[32] A.K. Kramosil, J. Michalek, Fuzzy metric and statistical metric spaces, Kybernetica 11 (1975), 326-334.

[33] V. Kumar, K Kumar, On the ideal convergence of sequences of fuzzy numbers, Inform. Sci. 178 (2008), 4670-4678.

[34] M. Mursaleen, S.A. Mohiuddine, Statistical convergence of double sequences in intuitionistic fuzzy normed spaces, Chaos Solitons and Fractals 41 (2009), 2414-2421.

[35] A. Nabin, S. Pehlivan, M. Gürdal, On J-Cauchy sequences, Taiwanese J. Math. 11:2 (2007), $569-576$.

[36] F. Nuray, E. Savaş, Statistical convergence of sequences of fuzzy numbers, Math. Slovaca 45 (1995), 269-273.

[37] W. Raymond, Y. Freese, J. Cho, Geometry of linear 2-normed spaces, N.Y. Nova Science Publishers, Huntington, 2001.

[38] E. Savaş, On statistically convergent sequence of fuzzy numbers, Inform. Sci. 137 (2001), $272-282$

[39] E. Savaş, On some new sequence spaces in 2-normed spaces using ideal convergence and an Orlicz function, J. Inequal. Appl. 2010 (2010), Art. ID 482392, 8 pp.

[40] E. Savaş, Some J-convergent sequence spaces of fuzzy numbers defined by infinite matrix, Math. Comp. Appl. 18:2 (2013), 84-93.

[41] C. Şençimen, S. Pehlivan, Statistical convergence in fuzzy normed linear spaces, Fuzzy Sets Syst. 159 (2008), 361-370.

[42] H. Steinhaus, Sur la convergence ordinaire et la convergence asymptotique, Colloq. Math. 2 (1951), 73-34. 
[43] R.M. Somasundaram, T. Beaula, Some aspects of 2-fuzzy 2-normed linear spaces, Bull. Malays. Math. Sci. Soc. (2) 32:2 (2009), 211-221.

[44] L.A. Zadeh, Fuzzy Sets, Inform. Control 8 (1965), 338-353.

[45] J. Zhang, The continuity and boundedness of fuzzy linear operators in fuzzy normed space, J. Fuzzy Math. 13:3 (2005), 519-536.

[46] A. Zygmund, Trigonometric Series, 2nd edition, Cambridge University Press, Cambridge, 1979. 